\title{
A PRODUÇÃO CIENTÍFICA SOBRE REPRESENTAÇÃO DE DADOS DENTRO DA ÁREA TEMÁTICA DA AGRICULTURA
}

\author{
LA PRODUCCIÓN CIENTÍFICA SOBRE \\ REPRESENTACIÓN DE DATOS DENTRO DEL ÁREA \\ TEMÁTICA DE LA AGRICULTURA
}

\author{
Jacquelin Teresa Camperos Reyes* \\ Ricardo César Gonçalves Sant'Ana** \\ Rogério Aparecido Sá Ramalho***
}

\begin{abstract}
RESUMO
Introdução: Este trabalho inquire sobre a relação existente entre a potencialidade agrícola da América Latina e a produção científica a partir de publicações da Ciência da Informação, a respeito da representação de dados na temática da agricultura. Estudos na área da representação de dados dentro de um setor da economia de relevância manifesta, como o da agricultura, revestem-se de importância por estabelecer pontes entre a ciência e o desenvolvimento de uma região ou mesmo um país. Objetivo: Analisar publicações do período de 2006 a 2016 sobre representação de dados, dentro do domínio da agricultura, a partir das instituições que os originaram, e a tendência dos conceitos neles abordados, com foco nos mecanismos XML e RDF. Metodologia: Desenvolveu-se pesquisa descritiva, bibliográfica, com abordagem qualitativa, identificando a dinâmica dos mecanismos XML e RDF, em periódicos da área da Ciência da Informação, na Base de Dados Referencial de Artigos de Periódicos em Ciência da Informação (BRAPCI), na base de dados Qualis da Comissão de Aperfeiçoamento de Pessoal do Nível Superior, e nos anais de eventos destacados na área da Ciência da Informação. Resultados: No universo dos trabalhos pesquisados foram identificadas 22 instituições, 13 localizadas na Europa e 1 na América Latina. Os conceitos delimitados nos trabalhos estudados decorrem no uso da XML e o RDF nesses dois tópicos: Interoperabilidade Semântica e Indexação automática. Conclusões: Os indícios levaram a percepção de uma situação

*Mestranda em Ciência da Informação Universidade Estadual Paulista - UNESP. Email: jcamperosreyes@gmail.com.

** Doutor em Ciência da Informação Livre-Docente em Sistemas de Informações Gerenciais Universidade Estadual Paulista - UNESP. E-mail: ricardosantana@marilia.unesp.br.

*** Doutor em Ciência da Informação Universidade Federal de São Carlos - UFSCar. E-mail: ramalho@ufscar.br.
\end{abstract}

Inf. Inf., Londrina, v. 22, n. 2, p. 467 - 480, maio/ago., 2017. http:www.uel.br/revistas/informacao/ 
Jacquelin Teresa Camperos Reyes, Ricardo César Gonçalves Sant'Ana, Rogério Aparecido Sá Ramalho

A produção científica sobre representação de dados dentro da área temática da agricultura

contraditória pela relação inversa entre a potencialidade agrícola da região latinoamericana e a produção científica no campo da representação de dados, neste caso, com estudos dos mecanismos XML e RDF. Espera-se coadjuvar na discussão em torno das contribuições científicas que a Ciência da Informação fornece, tendo como foco a representação de dados na temática da agricultura, com a intenção de consolidar a natureza social da Ciência da Informação e suas contribuições práticas.

Palavras-chave: Representação de dados. XML. RDF. Agricultura. América Latina.

\section{INTRODUÇÃO}

Inquire-se neste documento sobre a relação existente entre a potencialidade agrícola da América Latina e a produção científica a partir de publicações da Ciência da Informação, a respeito da representação de dados no domínio da agricultura. Observa-se o caráter social e material dos dados e as informações (FROHMANN, 2008), e o contributo, neste contexto, para a sociedade dos profissionais da Ciência da Informação como uma Ciência Social Aplicada (COMISSÃO DE APERFEIÇOAMENTO DE PESSOAL DO NÍVEL SUPERIOR, 2017).

No contexto da representação de dados e informações, Berners-Lee (2006) assevera que o formato básico para a interligação de dados é a dupla RDF (Resource Description Framework) / XML (Extensible Markup Language), sendo esses mecanismos, fundamentais para o intercâmbio de ativos informáticos na conjuntura da Internet.

O objetivo deste trabalho é analisar publicações do período de 2006 a 2016 sobre representação de dados, dentro do domínio da agricultura, a partir das instituições que os originaram e a tendência dos conceitos neles abordados, com foco nos mecanismos XML e RDF.

Para atingir o objetivo, desenvolveu-se uma pesquisa descritiva, bibliográfica, com abordagem qualitativa, em periódicos da área da Ciência da Informação na Base de Dados Referencial de Artigos de Periódicos em Ciência da Informação (BRAPCI), na base de dados Qualis da Comissão de Aperfeiçoamento de Pessoal do Nível Superior, e nos anais de eventos destacados na área da Ciência da Informação. Foram analisados escritos

Inf. Inf., Londrina, v. 22, n. 2, p. 467 - 480, maio/ago., 2017.

http:www.uel.br/revistas/informacao/ 
Jacquelin Teresa Camperos Reyes, Ricardo César Gonçalves Sant'Ana, Rogério Aparecido Sá Ramalho

A produção científica sobre representação de dados dentro da área temática da agricultura

científicos do período 2006 a 2016, identificando a dinâmica das tecnologias XML e RDF.

Estudos na área da representação de dados e informações dentro de um setor da economia de importância manifesta como o da agricultura revestem-se de importância por estabelecer pontes entre a ciência e o desenvolvimento de uma região ou mesmo um país. Espera-se contribuir na promoção da discussão em torno das contribuições científicas que a Ciência da Informação fornece, tendo como foco a representação de dados na temática da agricultura, com a intenção de consolidar a natureza social da Ciência da Informação e suas contribuições práticas.

Os indícios levaram à percepção de uma situação contraditória pela relação inversa entre a potencialidade agrícola da região latino-americana e a produção científica no campo da representação de dados, em este caso, com estudos dos mecanismos XML e RDF dentro do domínio da agricultura.

\section{COESÃO ENTRE ÁREAS TEMÁTICAS E OS ESTUdOS DE REPRESENTAÇÃO DE DADOS}

Diante do contexto tecnológico atual, ações para conciliar as práticas científicas dentro da área da ciência da informação com as práticas exercidas dentro das realidades sociais, políticas, económicas e culturais (FROHMANN, 2008), ganham ênfase crescente nas pesquisas na Ciência da Informação.

A sociedade contemporânea requer profissionais que pesquisem as realidades regionais, para que possam emergir pontes que enlacem a comunidade científica com o desenvolvimento e o crescimento integral da sociedade.

Países com perfil agrícola como o Brasil, por suas vantagens geográficas e socioeconômicas, tem impactadas positivamente suas economias pela renda econômica que esse setor fornece. O país produz não só o alimento para o consumo interno, como, ainda, atende a grande parte do que é consumido em outras partes do mundo.

Inf. Inf., Londrina, v. 22, n. 2, p. 467 - 480, maio/ago., 2017. http:www.uel.br/revistas/informacao/ 
Jacquelin Teresa Camperos Reyes, Ricardo César Gonçalves Sant'Ana, Rogério Aparecido Sá Ramalho

A produção científica sobre representação de dados dentro da área temática da agricultura

Segundo a Organização das Nações Unidas para a Alimentação e a Agricultura (FAO), e a Organização para a Cooperação e o Desenvolvimento Económico (OCDE) (2015), o Brasil é o segundo maior exportador agrícola do mundo, sendo o maior produtor de açúcar, suco de laranja e café.

Estudos de representação de dados e informações dentro do domínio da agricultura buscam subsidiar a partir da Ciência da Informação, o desenvolvimento socioeconômico por meio de pesquisas com fundamento conceitual levadas ao plano prático, buscando refletir em torno de um setor específico e tão significativo como o agrícola. Segundo o Instituto Brasileiro de Geografia e Estatística (IBGE) (2017), na quarta estimativa de 2017, para a safra nacional de cereais, houve um crescimento de $26,2 \%$ com respeito ao mesmo período do 2016, dado significativo considerando o impacto na economia do país, devido à relação direta com o emprego de mão de obra, fortalecimento de relações comerciais, até divisas por exportações.

Sendo assim, e diante das riquezas informacionais da internet, foram necessários mecanismos para estruturar recursos informacionais, que permitissem a sua representação, visando uma recuperação eficiente deles, ainda, dentro dos novos ambientes informacionais que estavam surgindo (SANTOS; ALVES, 2009).

O modelo de trabalho Resource Description Framework (RDF), desenvolvido pelo World Wide Web Consortium, fornece uma infraestrutura que permite a representação e a descrição de recursos informacionais na internet. RDF é um modelo independente da sintaxe, que possibilita a interoperabilidade de metadados, na representação de recursos, suas propriedades e relações (MILLER, 1998).

O RDF usa a linguagem Extensible Markup Language (XML). XML, também desenvolvido pelo World Wide Web Consortium, é um formato não proprietário que permite a criação de definições de dados, leitura humana do corpo do documento e a representação de estruturas de dados complexas. RDF e XML, com uma codificação consistente, têm a possibilidade de expressar semântica por meio de estruturas que representam dados e

Inf. Inf., Londrina, v. 22, n. 2, p. 467 - 480, maio/ago., 2017. 
Jacquelin Teresa Camperos Reyes, Ricardo César Gonçalves Sant'Ana, Rogério Aparecido Sá Ramalho

A produção científica sobre representação de dados dentro da área temática da agricultura

informações, aliás, com capacidades de intercâmbio e processamentos automáticos (MILLER, 1998).

\subsection{Método aplicado}

Este texto apresenta o resultado de uma pesquisa bibliográfica com abordagem qualitativa, que buscou a interpretação de fluxos nas produções científicas sobre representações de dados e informações com RDF e XML, dentro do domínio da agricultura.

A busca da literatura científica foi realizada em periódicos da área da Ciência da Informação na Base de Dados Referencial de Artigos de Periódicos em Ciência da Informação (BRAPCI), na base de dados Qualis ${ }^{1}$ em periódicos classificados como A1 e A2 da área de avaliação Comunicação e Informação e, nos anais de eventos destacados na área da Ciência da Informação: Encontro Nacional da Pesquisa em Ciência da Informação (ENANCIB) e das conferências do Dublin Core Metadata Initiative (DCMI).

Realizou-se a análise da literatura recuperada com os termos (XML e AGRICULTURA) e (RDF e AGRICULTURA) nos bancos de dados explicitados, delimitando o período de tempo de 2006 a 2016, abordando o conteúdo pleno dos trabalhos com o fim de identificar os fluxos das tecnologias XML e RDF dentro dos escopos de cada pesquisa.

Partindo das dinâmicas identificadas, foram delineadas as origens geográficas das instituições origem das publicações e uma categorização das aplicações que os autores têm estudado para os mecanismos XML e RDF. Em seguida, apresentam-se os resultados encontrados a partir dos documentos analisados, enquadrados na abordagem que os autores fazem desses mecanismos dentro do domínio da agricultura.

\footnotetext{
1 "[...] o conjunto de procedimentos utilizados pela Capes para estratificação da qualidade da
} produção intelectual dos programas de pós-graduação" (BRASIL, 2014).

Inf. Inf., Londrina, v. 22, n. 2, p. 467 - 480, maio/ago., 2017. 
Jacquelin Teresa Camperos Reyes, Ricardo César Gonçalves Sant'Ana, Rogério Aparecido Sá Ramalho

A produção científica sobre representação de dados dentro da área temática da agricultura

\section{ORIGEM DAS PRODUÇÕES E CONCEITOS IDENTIFICADOS}

Segundo cálculos realizados com dados do Banco Mundial (2014), a área agrícola dos países da América Latina é de $7.238 .400 \mathrm{~km}^{2}$, enquanto na Europa soma $4.072 .000 \mathrm{~km}^{2}$. Relacionando esses dados, encontra-se que a área agrícola europeia equivale apenas $56 \%$ da área agrícola do continente latino-americano.

Baseados em supostos macroeconómicos e projeções em políticas públicas, a Organização das Nações Unidas para a Alimentação e a Agricultura (FAO), e a Organização para a Cooperação e o Desenvolvimento Económico (OCDE) (2015) asseveram que a América Latina irá manter-se como o maior produtor neto de alimentos do mundo em uma perspectiva que vai até o ano 2023.

A figura 1 apresenta a distribuição geográfica das instituições que participaram das discussões analisadas neste estudo.

Figura 1 - Concentração geográfica origem das publicações

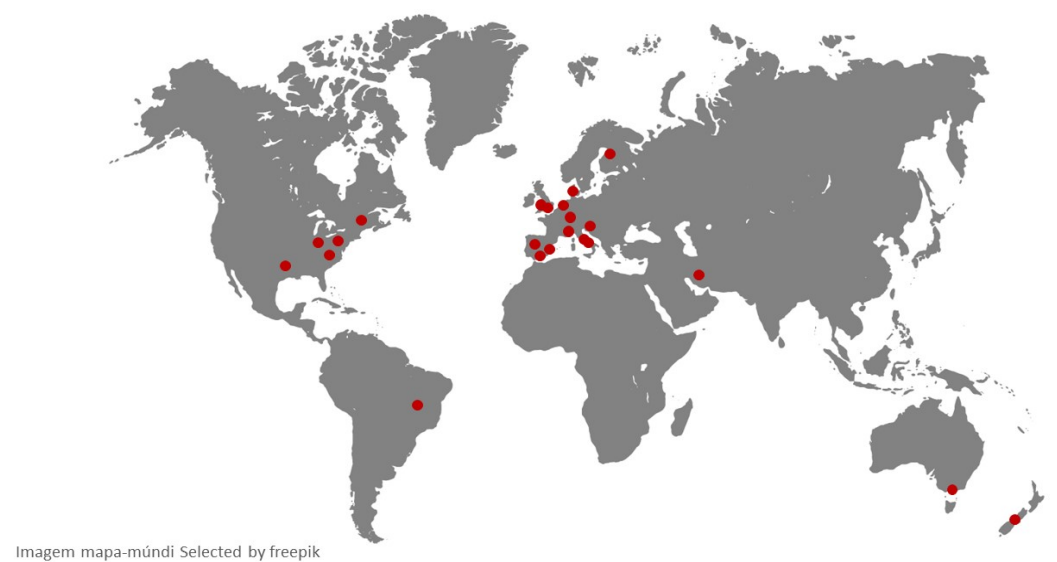

Fonte: Dados da pesquisa

No universo dos trabalhos pesquisados identificaram-se 22 instituições, 13 localizadas na Europa e 1 na América Latina, isto, em termos percentuais, significa que $59 \%$ dos trabalhos foram produzidos no antigo continente e $4,5 \%$ no latino-americano, especificamente em um trabalho originado na Empresa

Inf. Inf., Londrina, v. 22, n. 2, p. 467 - 480, maio/ago., 2017.

http:www.uel.br/revistas/informacao/ 
Jacquelin Teresa Camperos Reyes, Ricardo César Gonçalves Sant'Ana, Rogério Aparecido Sá Ramalho

A produção científica sobre representação de dados dentro da área temática da agricultura

Brasileira de Pesquisa Agropecuária (Embrapa) em parceria com o Institut National de la Recherche Agronomique (INRA) da França.

As instituições que originaram os trabalhos recuperados encontram-se no Quadro 1.

Quadro 1 - Instituições participantes e o seu país de origem

\begin{tabular}{|c|c|}
\hline INSTITUIÇÃO & PAís \\
\hline \multicolumn{2}{|c|}{ Natureza Pública } \\
\hline FAO & Organização de nações \\
\hline Universidad Carlos III de Madrid & Espanha \\
\hline University of Melbourne & Austrália \\
\hline Universidad de Murcia & Espanha \\
\hline Concordia University & Canadá \\
\hline $\begin{array}{l}\text { Institut national de la recherche } \\
\text { agronomique (INRA) }\end{array}$ & França \\
\hline $\begin{array}{l}\text { Empresa Brasileira de Pesquisa } \\
\text { Agropecuária (EMBRAPA) }\end{array}$ & Brasil \\
\hline University of Waikato & Nova Zelândia \\
\hline Universidad Politécnica de Valencia & Espanha \\
\hline University of Tor Vergata & Itália \\
\hline $\begin{array}{l}\text { Leibniz-Institute for the Social Sciences } \\
\text { (GESIS) }\end{array}$ & Alemanha \\
\hline $\begin{array}{l}\text { Iran Regional Information Center for } \\
\text { Science and Technology }\end{array}$ & Irã \\
\hline University of Bath & Reino Unido \\
\hline University of South Wales & Reino Unido \\
\hline Kent State University & Estados Unidos \\
\hline University of Ljubljana & Eslovênia \\
\hline
\end{tabular}

Inf. Inf., Londrina, v. 22, n. 2, p. 467 - 480, maio/ago., 2017. http:www.uel.br/revistas/informacao/ 
Jacquelin Teresa Camperos Reyes, Ricardo César Gonçalves Sant'Ana, Rogério Aparecido Sá Ramalho

A produção científica sobre representação de dados dentro da área temática da agricultura

\begin{tabular}{|l|l|}
\hline University of Illinois at Urbana Champaign & Estados Unidos \\
\hline University of North Carolina & Estados Unidos \\
\hline University of Denmark & Dinamarca \\
\hline University of Oulu & Finlândia \\
\hline \multicolumn{2}{|c|}{ Natureza Privada } \\
\hline PEROT SYSTEMS Natureza Mista \\
\hline \multicolumn{2}{|c|}{ Estados Unidos } \\
\hline University Vrije & Holanda \\
\hline
\end{tabular}

Fonte: dados da pesquisa

Nas instituições listadas, $91 \%$ são de natureza pública, e $73 \%$ universidades. Destaca-se a participação dos centros de pesquisa: o LeibnizInstitute for the Social Sciences (GESIS) da Alemanha, o Institut national de la recherche agronomique (INRA) da França, o Iran Regional Information Center for Science and Technology, e a Empresa Brasileira de Pesquisa Agropecuária (EMBRAPA). O GESIS apresenta uma organização interessante pelo fato de que os membros, tanto do Conselho de Curadores quanto do Conselho Consultivo Científico, provêm de um conjunto de órgãos de governo e instituições universitárias (11 universidades da Alemanha, 1 da Suíça, 1 da Áustria, e, 1 do Reino Unido). Mais de 50 \% dos membros dos conselhos provêm de instituições de ensino superior, o que denota a importância que para a Alemanha representa a parceria entre os estudos acadêmicos e as políticas públicas como pontos chaves no desenvolvimento do país.

Nos documentos analisados foram observados 48 autores distintos, 13 deles vinculados com a FAO, nenhum dos 13 provenientes da América Latina. Um autor da FAO concentra a coautoria em sete dos 20 artigos científicos estudados. Dois autores associados com a EMBRAPA, 4\% do conjunto de autores, representam o continente latino-americano no escopo desta pesquisa.

Esta análise de produção científica no domínio da agricultura referente aos mecanismos XML e RDF apresenta uma relação inversa entre o grande potencial agrícola dos países da América Latina e a quantidade de discussões

Inf. Inf., Londrina, v. 22, n. 2, p. 467 - 480, maio/ago., 2017. http:www.uel.br/revistas/informacao/ 
Jacquelin Teresa Camperos Reyes, Ricardo César Gonçalves Sant'Ana, Rogério Aparecido Sá Ramalho

A produção científica sobre representação de dados dentro da área temática da agricultura

dessas tecnologias que sustentam a representação de dados e informações com os mecanismos XML e RDF.

É um assunto que poderia observar-se como contraditório, a realidade de que o continente latino-americano tenha uma liderança na produção neta de alimentos em nível global, e que no escopo desta pesquisa, tenha se identificado só uma reflexão em torno ao domínio da agricultura a partir do olhar de uma ciência social aplicada como o da Ciência da Informação.

Abre-se porta à questões sobre o direcionamento de pesquisas a partir da área de atuação da Ciência da Informação, ainda pelo fato da relação que o Brasil tem com profissionais formando-se em programas de pós-graduação, que são originários de outros países latino-americanos.

Com relação aos conceitos identificados nos trabalhos estudados, os autores decorrem dentro do uso da XML e o RDF nessas tendências: a busca de Interoperabilidade Semântica, e, processos para realizar Indexação Automática utilizando tesauros.

O Quadro 2 apresenta os tópicos e subtópicos objetos das discussões com as frequências observadas.

Quadro 2 - Tópicos e frequências dos assuntos nas pesquisas

\begin{tabular}{|c|l|c|}
\hline Tópicos & \multicolumn{1}{|c|}{ Subtópicos } & Frequência \\
\hline \multirow{4}{*}{$\begin{array}{c}\text { Interoperabilidade } \\
\text { Semântica }\end{array}$} & $\begin{array}{l}\text { Mapeamento de tesauros } \\
\text { o Linked Open Data (LOD) }\end{array}$ & 6 \\
\cline { 2 - 3 } & $\begin{array}{l}\text { Implementação de } \\
\text { Ontologias }\end{array}$ & 6 \\
\cline { 2 - 3 } & $\begin{array}{l}\text { Desambiguação de } \\
\text { metadados }\end{array}$ & 1 \\
\hline \multirow{2}{*}{$\begin{array}{l}\text { Indexação } \\
\text { automática }\end{array}$} & $\begin{array}{l}\text { Estimações no uso } \\
\text { alternativo de tesauros para } \\
\text { indexação automática }\end{array}$ & 3 \\
\hline
\end{tabular}

Fonte: dados da pesquisa

Inf. Inf., Londrina, v. 22, n. 2, p. 467 - 480, maio/ago., 2017. http:www.uel.br/revistas/informacao/ 
Jacquelin Teresa Camperos Reyes, Ricardo César Gonçalves Sant'Ana, Rogério Aparecido Sá Ramalho

A produção científica sobre representação de dados dentro da área temática da agricultura

Promover a discussão dessas categorias identificadas, especialmente focadas na Interoperabilidade Semântica, poderia contribuir com o desenvolvimento agrícola dos países latino-americanos? Ainda, sendo na atualidade líderes mundiais na produção de alimentos, qual seria o contributo ao incrementar as pesquisas no campo da representação de dados e informações partindo da Ciência da Informação?

A Ciência da Informação ao desenvolver-se tanto como ciência pura, quanto aplicada na geração de produtos e serviços (BORKO, 1968), aborda as questões relacionadas com a informação, suas estruturas e seus processos (SARACEVIC, 1996). A pesquisa realizada partindo da Ciência da Informação, tenta aplicar as teorias da informação, teorias das decisões, construtos da ciência cognitiva, da lógica, dentre outras, aplicadas todas com o rigor científico integrante (SARACEVIC, 1996).

Contudo, fundamentando-se no conceito do Saracevic (1996, p.47) que afirma que:

\begin{abstract}
A Ciência da Informação é um campo dedicado às questões científicas e à prática profissional voltadas para os problemas da efetiva comunicação do conhecimento e de seus registros entre os seres humanos, no contexto social, institucional ou individual do uso e das necessidades de informação.
\end{abstract}

O incremento das pesquisas dentro do domínio da agricultura, a partir da perspectiva e as possibilidades científicas da Ciência da Informação, deveria contribuir com efetividade permitindo que os atores demandantes das análises, reflexões, propostas, serviços e processos, possam atender às suas necessidades informacionais.

A missão do Leibniz-Institute for the Social Sciences GESIS (2017) expõe que, ela é uma aliança de instituições de pesquisa e infraestrutura das Ciências Sociais, ligadas ao princípio "theoria cum praxi - Utilizar a ciência para o bem do povo".

\title{
4 CONSIDERAÇÕES FINAIS
}

Tendo em conta a atual transição da web de documentos para a web de dados, estudos focados em domínios de conhecimento específicos, como o da

Inf. Inf., Londrina, v. 22, n. 2, p. 467 - 480, maio/ago., 2017.

http:www.uel.br/revistas/informacao/ 
Jacquelin Teresa Camperos Reyes, Ricardo César Gonçalves Sant'Ana, Rogério Aparecido Sá Ramalho

A produção científica sobre representação de dados dentro da área temática da agricultura

agricultura, contribuem na consolidação da Ciência da Informação como área essencial para o desenvolvimento tanto econômico quanto social nas regiões, por meio de projetos que propõem o uso de tecnologias que auxiliam a dinâmica dos dados.

A potencialidade agrícola do conglomerado de países latino-americanos, espera contribuições dos pesquisadores em processos que envolvem dados, no tratamento digital deles, projetando estudos e implementações que possam surgir a partir da Ciência da Informação, e que sejam estratégicos para robustecer setores econômicos de importância vital no nível mundial, como é a produção de alimentos.

Propõem-se futuras reflexões multidisciplinares, apoiadas nos profissionais da Ciência da Informação, dentro da temática da agricultura na América Latina e o uso de mecanismos basilares para a interoperabilidade semântica, como são a XML e o RDF. Alguns dos tópicos a analisar poderiam estar relacionados com: a influência direta na produtividade da agricultura do reduzido acompanhamento da comunidade científica, especificamente dos cientistas da informação; a aplicação de teorias científicas para a tomada de decisões, baseadas em coleta de dados estruturados na web de dados; otimização da gestão dos ativos informáticos dos países da região, na busca de melhorias conjunturais no domínio da agricultura, com a utilização de mecanismos para a representação e descrição de dados como o RDF e a XML.

Agradecimentos: Organização dos Estados Americanos (OEA).

\section{REFERÊNCIAS}

BANCO MUNDIAL. Datos. [2014]. Disponível em:

<https://datos.bancomundial.org/indicator/AG.LND.AGRI.ZS>. Acesso em: 05 abr. 2017.

BERNERS-LEE, T. Linked data. 2006. Disponível em:

<https://www.w3.org/Designlssues/LinkedData.html>. Acesso em: 08 mai. 2017.

Inf. Inf., Londrina, v. 22, n. 2, p. 467 - 480, maio/ago., 2017.

http:www.uel.br/revistas/informacao/ 
Jacquelin Teresa Camperos Reyes, Ricardo César Gonçalves Sant'Ana, Rogério Aparecido Sá Ramalho

A produção científica sobre representação de dados dentro da área temática da agricultura

BORKO, H. Information science: what is it? American Documentation, v. 19, n. 1, p. 3-5, 1968. Disponível em: <http://skat.ihmc.us/rid=1KR7TR64BNMXB04-5SVP/BORKO(1968)-533107-Borko-H-v-19-n-1-p-35-1968.pdf\%3E>. Acesso em: 13 mai. 2017

BRASIL. Ministério da Educação. Fundação Capes: Qualis. 2014. Disponível em: <http://www.capes.gov.br/component/content/article?id=2550: capesaprova-a-nova-classificacao-do-qualis>. Acesso em: 10 mai. 2017.

COMISSÃO DE APERFEIÇOAMENTO DE PESSOAL DO NÍVEL SUPERIOR (CAPES). Áreas de conhecimento. 2017. Disponível em:

<https://sucupira.capes.gov.br/sucupira/public/index.jsf>. Acesso em: 10 de mai. 2017

FROHMANN, B. O caráter social, material e público da Informação. In: FUJITA, M. S. L; MARTELETO, R. M.; LARA, M. L. G. de. (Org.). A Dimensão

Epistemológica da Ciência da Informação e suas Interfaces Técnicas, Políticas e Institucionais nos Processos de Produção, Acesso e

Disseminação da Informação. São Paulo: Cultura Acadêmica Editora; Marília: Fundepe Editora, 2008. p. 19-34.

INSTITUTO BRASILEIRO DE GEOGRAFIA E ESTATÍSTICA. Sala de Imprensa. 2017. Disponível em: < https://agenciadenoticias.ibge.gov.br/>. Acesso em: 16 de mai. 2017.

LEIBNIZ-INSTITUTE FOR THE SOCIAL SCIENCES (GESIS). GESIS Mission Statement. 2017. Disponível em: <https://www.gesis.org/en/institute/theassociation/mission/>. Acesso em: 15 mai. 2017.

MILLER, E. An Introduction to the Resource Description Framework. D-Lib Magazine, v. 4, n. 5, 1998. Disponível em:

<http://www.dlib.org/dlib/may98/miller/05miller.html>. Acesso em: 10 mai. 2017.

ORGANIZAÇÃO DAS NAÇÕES UNIDAS PARA A ALIMENTAÇÃO E A

AGRICULTURA; ORGANIZAÇÃO PARA A COOPERAÇÃO E O

DESENVOLVIMENTO ECONÔMICO. Perspectivas Agrícolas 2015. 2015.

OECD Publishing, Paris.

SANTOS, P. L. V. A. da C.; ALVES, R. C. V. Metadados e Web Semântica para estruturação da Web 2.0 e Web 3.0. DataGramaZero, Rio de Janeiro, v. 10, n. 6, dez. 2009.

SARACEVIC, T. Ciência da informação: origem, evolução e relações.

Perspectivas em ciência da informação, Belo Horizonte, v. 1, n. 1, p. 41-62, jan./jun. 1996. Disponível em:

$<$ http://portaldeperiodicos.eci.ufmg.br/index.php/pci/article/view/235>. Acesso em: 12 mai. 2017.

Title

Inf. Inf., Londrina, v. 22, n. 2, p. 467 - 480, maio/ago., 2017. 
Jacquelin Teresa Camperos Reyes, Ricardo César Gonçalves Sant'Ana, Rogério Aparecido Sá Ramalho

A produção científica sobre representação de dados dentro da área temática da agricultura

The scientific production on data representation within the thematic area of agriculture

\begin{abstract}
Introduction: This document inquiries about the relation between the agricultural potential of Latin America and the scientific production from publications of Information Science, as respects to the data representation in the agriculture area. Studies in the data representation area within a sector of the economy of manifest relevance, as for the agricultural, deal with the importance of establishing bridges among the science and the development of a region or even a country. Objective: The objective is to analyze publications from 2006 to 2016 related to data representation, inside the agriculture domain, starting from the institutions that originated them, and the tendency of the concepts approached by them, with focal point in XML and RDF mechanisms. Methodology: A descriptive, bibliographic, with a qualitative approach, research was developed, identifying the dynamic of the XML and RDF mechanisms, in journals of the Information Science area, in the Referential Database of Articles of Journals in Information Science (BRAPCI), in the Qualis database of the Comissão de Aperfeiçoamento de Pessoal do Nível Superior, and the noteworthy conference proceedings in the Information Science area. Results: There were 22 institutions identified in the sphere of the examined documents, 13 of them localized in Europe and 1 in Latin America. The delimitated concepts in the studied works move onward the use of XML and RDF in these two topics: Semantic Interoperability and automatic Indexation. Conclusions: The indications led to the perception of a contradictory situation by the inverse relation between the agricultural potential of the Latin-American region and the scientific production in the data representation field, in this case, with studies of XML and RDF mechanisms. It is expected to collaborate in the discussion around scientific contributions that Information Science provides, focusing on data representation in the agriculture domain, with the intention of consolidating the social nature of Information Science and its practical contributions.
\end{abstract}

Keywords: Data representation. XML. RDF. Agriculture. Latin America.

\title{
Título
}

La producción científica sobre representación de datos dentro del área temática de la agricultura

\section{Resumen}

Introducción: Este trabajo inquiere sobre la relación existente entre la potencialidad agrícola de América Latina y la producción científica a partir de publicaciones de la Ciencia de la Información, a respecto de la representación de datos en la temática de la agricultura. Estudios en el área de la representación de datos dentro de un sector de la economía de relevancia manifiesta, como el de la agricultura, se revisten de importancia por establecer puentes entre la ciencia y el desarrollo de una región o incluso un país. Objetivo: Analizar publicaciones del período 2006 a 2016 sobre representación de datos, dentro del dominio de la agricultura, a partir de las instituciones que los originaron, y la tendencia de los conceptos en ellos abordados, focalizando en los mecanismos XML e RDF. Metodología: Se desarrolló investigación descriptiva, bibliográfica, con abordaje cualitativo, identificando la dinámica de los mecanismos XML e RDF, en periódicos del área de la Ciencia de la Informação, en la Base de Datos Referencial de Artículos de Periódicos en Ciencia de la Información

Inf. Inf., Londrina, v. 22, n. 2, p. 467 - 480, maio/ago., 2017. 
Jacquelin Teresa Camperos Reyes, Ricardo César Gonçalves Sant'Ana, Rogério Aparecido Sá Ramalho

A produção científica sobre representação de dados dentro da área temática da agricultura

(BRAPCI), en la base de dados Qualis de la Comissão de Aperfeiçoamento de Pessoal do Nível Superior, y en las memorias de eventos destacados en el área de la Ciencia de la Información. Resultados: En el universo de los trabajos investigados fueron identificadas 22 instituciones, 13 localizadas en Europa y 1 en América Latina. Los conceptos delimitados en los trabajos estudiados discuten el uso de XML y RDF en estos dos tópicos: Interoperabilidad Semántica e Indexación automática. Conclusiones: Los indicios llevaron a la percepción de una situación contradictoria por la relación inversa entre la potencialidad agrícola de la región latino-americana y la producción científica en el campo de la representación de datos, en este caso, con estudios de los mecanismos XML e RDF. Se espera coadyuvar en la discusión en torno de las contribuciones científicas que la Ciencia de la Información provee, teniendo como foco la representación de datos en la temática de la agricultura, con la intención de consolidar la naturaleza social de Ciencia de la Información y sus contribuciones prácticas.

Palabras clave: Representación de datos. XML. RDF. Agricultura. América Latina.

Recebido: 30.08 .2017

Aceito: 30.09 .2017

Inf. Inf., Londrina, v. 22, n. 2, p. 467 - 480, maio/ago., 2017. 